\title{
Restoration of Guyton's Diagram for Regulation of the Circulation as a Basis for Quantitative Physiological Model Development
}

\author{
J. KOFRÁNEK ${ }^{1}$, J. RUSZ ${ }^{1,2}$ \\ ${ }^{1}$ Laboratory of Biocybernetics, Department of Pathophysiology, First Faculty of Medicine, Charles \\ University in Prague, Czech Republic, ${ }^{2}$ Department of Circuit Theory, Faculty of Electrical \\ Engineering, Czech Technical University in Prague, Czech Republic
}

Received June 25, 2009

Accepted April 16, 2010

On-line June 9, 2010

\begin{abstract}
Summary
We present the current state of complex circulatory dynamics model development based on Guyton's famous diagram. The aim is to provide an open-source model that will allow the simulation of a number of pathological conditions on a virtual patient including cardiac, respiratory, and kidney failure. The model will also simulate the therapeutic influence of various drugs, infusions of electrolytes, blood transfusion, etc. As a current result of implementation, we describe a core model of human physiology targeting the systemic circulation, arterial pressure and body fluid regulation, including short- and long-term regulations. The model can be used for educational purposes and general reflection on physiological regulation in pathogenesis of various diseases.
\end{abstract}

\section{Key words}

Body fluid homeostasis - Blood pressure regulation • Physiological modeling • Guyton's diagram

\section{Corresponding author}

J. Rusz, Department of Circuit Theory, Faculty of Electrical Engineering, Czech Technical University, Technická 2, 16627 Prague 6, Czech Republic. E-mail: ruszjan@fel.cvut.cz

\section{Introduction}

The landmark achievement closely associated with integrative physiology development was the circulatory dynamics model published by Prof. Arthur C. Guyton and his collaborators (Guyton et al. 1972). Subsequently, its more detailed description was published in the monograph one year later (Guyton et al. 1973).
This model represents the first large-scale mathematical description of interconnected physiological subsystems of the body. The model was described by a sophisticated graphic diagram with various computing blocks symbolizing quantitative physiological feedback connections. The diagram was published as a picture and the actual realization of the model was implemented in the FORTRAN language.

Although the FORTRAN implementation worked correctly, the diagram contains a number of errors that cause wrong model behavior. Moreover, FORTRAN implementation is not in agreement with this famous graphic diagram, it is almost unavailable nowadays, and contains several programming and computation-related features that require special treatment (Thomas et al. 2008). Despite the fact that the model was published over 30 years ago, it is currently used as a base for a number of research studies in the field of physiology (Montani and Van Vliet 2009, Osborn et al. 2009) and physiological modeling (Hunter et al. 2002, Bassingthwaighte 2000, 2009, Thomas et al. 2008), including research on the physiological consequences of weightlessness in manned space flight (White et al. 1991, 2003), or in a new approach to automation in medicine (Nguyen et al. 2008). In addition, the diagram is still reprinted with the original errors (Hall 2004, Bruce and Montani 2005). The overall revision of the diagram requires exhaustive search for errors and sophisticated analyses of physiological regulations system.

Here, we present a prototype of a core model of human physiology based on the original Guyton's diagram targeting the short- and long-term regulation of 


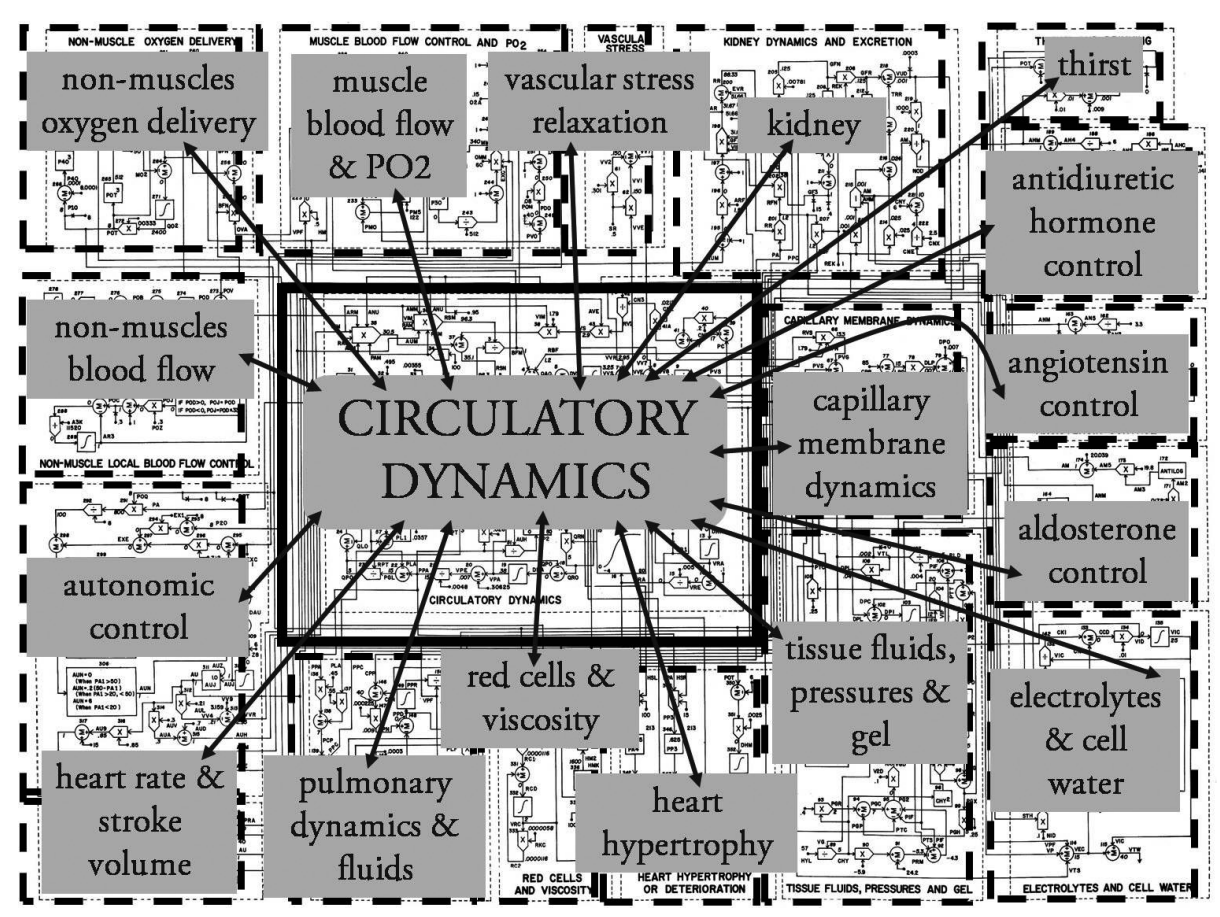

Fig. 1. Block diagram of the original Guyton's model subscribed by subsystems.

blood pressure, body fluids and homeostasis of the major solutes. This model also includes the hormonal (antidiuretic hormone, aldosterone and angiotensin) and nervous regulators (autonomic control), and the main regulatory sensors (baro- and chemoreceptors). Our complex circulatory dynamics model corresponds to the same graphic notation of the original Guyton's diagram and adheres to its basic physiological principles. While new models are continuously being developed (Srinivasan et al. 1996, Abram et al. 2007, Hester et al. 2008), our model finally brings a fully functional modification of the original Guyton's diagram, which is more suitable for a better and deeper understanding of the importance of physiological regulations and their use in development of many pathophysiological conditions by using simulation experiments.

The resulting model can be used as a baseline for the quantitative physiological model development designated for physicians' e-learning and acute care medicine simulators. This model can also be used as an effective learning aid for physiological regulation systems education, connected with biomedical engineering specialization. The model is provided as an open-source and it is downloadable at $<$ http://physiome.cz/guyton/>.

\section{Methods}

Mathematical model of global physiological regulation of blood pressure

The model consists of 18 modules containing approximately 160 variables and including 36 state variables (see Table 1 for more details). Each module represents an interconnected physiological subsystem (kidney, tissue fluid, electrolytes, autonomous nervous regulation and hormonal control including antidiuretic hormone, angiotensin and aldosterone). The model is constructed around a 'central' circulatory dynamics module in interaction with 17 'peripheral' modules corresponding to physiological functions (Fig. 1) and complete model targeting the systemic circulation, arterial pressure and body fluid regulation, including short- and long-term regulations. A graphic presentation of the model allows a display of the connectivity among all physiological relationships. In essence, the model contains a total of approximately 500 numerical entities (model variables, parameters and constants). Members of the original Guyton's laboratory have been continuously developing a more sophisticated version of the model, which is used for teaching (Abram et al. 2007). Although it includes about 4000 variables, this more elaborate model is less suited for our purposes than the model by Guyton et al. (1972), because of its incomplete description and physiological relationships formulation.

\section{Physiological regulations system analyses}

The original model represented as a sophisticated graphic diagram contains a number of errors which imply entirely incorrect physiological model behavior. The correction of these errors demanded complicated physiological regulations system analyses. 
Table 1. List of state variables used in the original Guyton's diagram with physiological significances, block numbers, and abbreviations.

\begin{tabular}{|c|c|c|}
\hline State variable in selected subsystem & Block number & Abbreviation \\
\hline Circulatory dynamics & $1-60$ & - \\
\hline 01. Venous vascular volume & 6 & VVS \\
\hline 02. Right atrial volume & 13 & VRA \\
\hline 03. Volume in pulmonary arteries & 19 & VPA \\
\hline 04. Volume in left atrium & 25 & VLA \\
\hline 05. Volume in systemic arteries & 31 & VAS \\
\hline Vascular stress relaxation & $61-65$ & - \\
\hline 06. Increased vascular volume caused by stress relaxation & 65 & VV7 \\
\hline Capillary membrane dynamics & $66-82$ & - \\
\hline 07. Plasma volume & 71 & VP \\
\hline 08. Total plasma protein & 80 & PRP \\
\hline Tissue fluids, pressure and gel & $83-113$ & - \\
\hline 09. Total interstitial fluid volume & 84 & VTS \\
\hline 10. Volume of interstitial fluid gel & 101 & VG \\
\hline 11. Interstitial fluid protein & 103 & IFP \\
\hline 12. Total protein in gel & 112 & GPR \\
\hline Electrolytes and cell water & $114-135$ & - \\
\hline 13. Total extracellular sodium & 118 & NAE \\
\hline 14. Total extracellular fluid potassium & 122 & $\mathrm{KE}$ \\
\hline 15. Total intracellular potassium concentration & 131 & KI \\
\hline Pulmonary dynamics and fluids & $136-152$ & - \\
\hline 16. Pulmonary free fluid volume & 142 & VPF \\
\hline 17. Total protein in pulmonary fluids & 149 & PPR \\
\hline Angiotensin control & $153-163$ & - \\
\hline 18. Angiotensin concentration & 159 & ANC \\
\hline Aldosterone control & $164-174$ & - \\
\hline 19. Aldosterone concentration & 170 & AMC \\
\hline Antidiuretic hormone control & $175-189$ & - \\
\hline 20. Degree of adaption of the right atrial pressure & 180 & AHY \\
\hline 21. Antidiuretic hormone concentration & 185 & $\mathrm{AHC}$ \\
\hline Thirst and drinking & $190-194$ & - \\
\hline Kidney dynamics and excretion & $195-222$ & - \\
\hline Muscle blood flow control and $\mathrm{PO}_{2}$ & $223-254$ & - \\
\hline 22. Rate of increase in venous vascular volume & 231 & DVS \\
\hline 23. Total volume of oxygen in muscle cells & 238 & QOM \\
\hline 24. Muscle vascular constriction caused by local tissue control & 254 & AMM \\
\hline Non-muscle oxygen delivery & $255-272$ & - \\
\hline 25. Non-muscle venous oxygen saturation & 260 & OSV \\
\hline 26. Non-muscle total cellular oxygen & 271 & QO2 \\
\hline Non-muscle, non-renal local blood flow control & $273-290$ & - \\
\hline 27. Vasoconstrictor effects of rapid autoregulation & 278 & AR1 \\
\hline 28. Vasoconstrictor effects of intermediate autoregulation & 285 & AR2 \\
\hline 29. Vasoconstrictor effects of long-term autoregulation & 289 & AR3 \\
\hline Autonomic control & $291-320$ & - \\
\hline 30. Time delay for realization of autonomic drive & 305 & AU4 \\
\hline
\end{tabular}


31. Overall activity of autonomic system

\section{Heart rate and stroke volume}

Red cells and viscosity

32. Volume of red blood cells

33. Hematocrit

Hearth hypertrophy or deterioration

34. Hypertrophy effect on left ventricle

35. Hypertrophy effect on heart

36. Cardiac depressant effect of hypoxia
310

AUJ

321 - 328

329 - 339

332

VRC

336

HM2

329 - 352

344

HPL

349

HPR

352

HMD

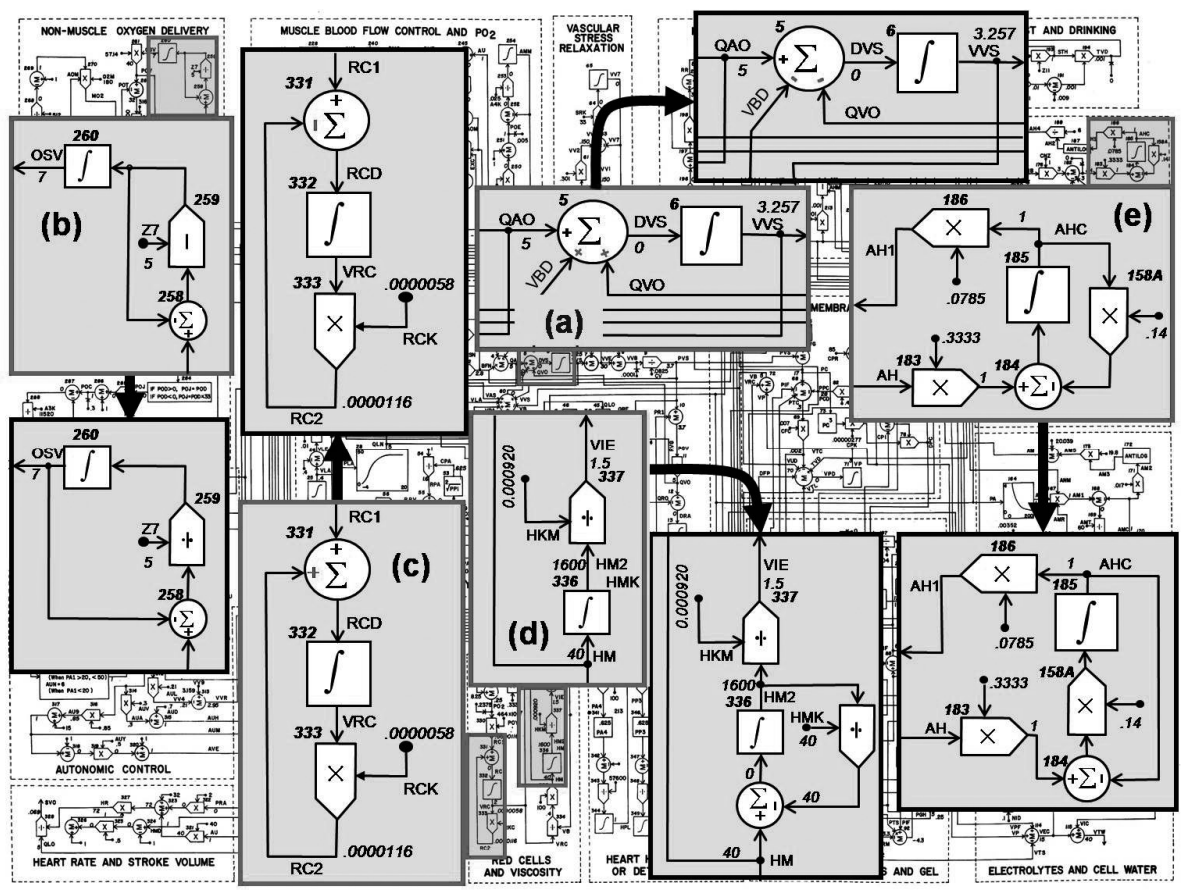

Fig. 2. The most significant errors of the original diagram and their correction.

These include exhaustive revision of the complete model and its behavior validation using several simulation experiments. In this stage, the original FORTRAN code of the Guyton's model was also used to compare the obtained simulation results. It is of course the case that the original FORTRAN code runs correctly; the errors were only in the diagram.

Because it would be beyond the scope of this paper to discuss each error in the original Guyton's diagram, as an example of the system analyses, we describe the five most significant errors which would have the greatest role in creating the unpredictable model behavior (Fig. 2). The other errors are mostly caused by replaced mathematical operations, wrong set of normalization and damping constants, and replaced signs that determine the positive or negative feedback.

The first error is the wrong flow direction marking of blood flow in the circulatory dynamics subsystem (Fig. 2a). The rate of increase in systemic venous vascular blood volume (DVS) is the subtraction between all rates of inflows and rates of outflows. Blood flow from the systemic arterial system (QAO) means inflow and rate from veins into the right atrium (QVO) means outflow. Rate change of the vascular system filling as the blood volume changes (VBD) is calculated as the difference between the summation of vascular blood compartments and blood volume overall capacity, meaning that VBD is found in the outflow rate too. Equation (1) gives DVS:

Correct eq.: $D V S=Q A O-V B D-Q V O$,
Erroneous eq.: $D V S=Q A O+V B D+Q V O$.

The second error is an algebraic loop in the nonmuscle oxygen delivery subsystem (Fig. 2b). There is a wrong feedback connection in venous oxygen saturation (OSV), which would cause a constant rise of OSV and the model would rapidly became unstable. Equation (2) 
gives the OSV from the blood flow in non-renal, nonmuscle tissues (BFN), oxygen volume in aortic blood (OVA), rate of oxygen delivery to non-muscle cells (DOB) and hematocrit (HM).

Correct eq.:

$\frac{d(O S V)}{d t}=\left(\frac{B F N \times O V A-D O B}{B F N \times H M \times 5}-O S V\right) / Z 7$,

Erroneous eq.:

$\frac{d(O S V)}{d t}=\frac{B F N \times O V A-D O B}{B F N \times H M \times 5}-\frac{B F N \times O V A-D O B}{B F N \times H M \times 5 \times Z 7}$.

Errors 3 and 4 involve simple subsystem red cells and viscosity. The third one is caused by positive feedback in the volume of red blood cells (VRC) computation (see Fig. 2c). Equation (3) gives the VRC from the red cell mass production rate $(\mathrm{RC} 1)$ and rate factor for red cells destruction (RCK) where the product between VRC and RCK gives the red cell mass destruction rate,

Correct eq.: $\frac{d(V R C)}{d t}=R C 1-V R C \times R C K$,

Erroneous eq.: $\frac{d(V R C)}{d t}=R C 1+V R C \times R C K$.

The fourth error is caused by a missing negative feedback in the portion of blood viscosity caused by red blood cells (VIE) computation (Fig. 2d). VIE is computed from the output of integrator HM2 (HM after integration divided by the normalization parameter HKM). Without the negative feedback, HM2 would incessantly rise. Viscosity is proportionate to hematocrit and the integrator acts as a dampening element in the original Guyton's model. From experimental data it can be derived that dependence of blood viscosity on hematocrit is not linearly proportional (Guyton et al. 1973). In equation (4), we designed a negative feedback by adding HMK constant into the feedback and by changing the HKM normalization parameter, which caused stabilized behavior of HM2,

Correct eq.: $\frac{d(H M 2)}{d t}=H M-\frac{H M 2}{H M K}$,

Erroneous eq.: $\frac{d(H M 2)}{d t}=H M$.
The fifth error is in the antidiuretic hormone control subsystem. The problem is in normalized antidiuretic hormone control computation (AHC) and computation of normalized rate of antidiuretic hormone creation $(\mathrm{AH} \times 0.3333)($ Fig. $2 \mathrm{e})$, when both values have a value of 1 under normal conditions. The solution emerges from the classic compartment approach. The hormone inflows into the whole-body compartment at the rate $F_{I}$ and outflows at the rate $F_{O}$. Rate of its depletion is proportional to its concentration $\mathrm{c}$, where $\mathrm{F}_{\mathrm{O}}=\mathrm{k} \times \mathrm{c}$, and concentration depends on overall quantity of hormone $\mathrm{M}$ and on capacity of distribution area V. Equation (5) gives the quantity of hormone $\mathrm{M}$ in whole-body compartment, which depends on the balance between hormone inflow and outflow,

$$
\frac{d M}{d t}=F_{I}-\frac{k M}{V} .
$$

Provided that the capacity of distribution area $\mathrm{V}$ is constant, we will substitute the ratio $\mathrm{k} / \mathrm{V}$ with constant $\mathrm{k}_{1}$. Guyton calculated the concentration of hormone $c_{0}$ normalized as a ratio of current concentration $c$ to its normal value $\mathrm{c}_{\text {norm }}=\mathrm{c} / \mathrm{c}_{0}$. At invariable distribution area $\mathrm{V}$, ratio of concentrations is the same as a ratio of current hormone overall quantity $\mathrm{M}$ to overall hormone quantity under normal conditions $\mathrm{M}_{\text {norm }}=\mathrm{M} / \mathrm{c}_{0}$. When we formulate the rate of flow in a normalized way (as a ratio to normal rate), under normal conditions it holds that $\mathrm{F}_{\mathrm{I}}=1, \mathrm{dM}_{\text {norm }} / \mathrm{dt}=0$ and after substituting it into equation (5) we get the equation (6),

$$
1-k_{1} M_{\text {norm }}=0 \text {. }
$$

The relative concentration of hormone $\mathrm{c}_{0}$ can be formulated as equation (7),

$$
c_{0}=\frac{M}{M_{\text {norm }}}=k_{1} M,
$$

and after final adjustments and inserting into a differential equation (7) we arrive at

$$
\begin{aligned}
& \text { Correct eq.: } \frac{d c_{0}}{d t}=\left(F_{I}-c_{0}\right) k_{1}, \\
& \text { Erroneous eq.: } \frac{d c_{0}}{d t}=F_{I}-c_{0} k_{1} .
\end{aligned}
$$


According to equation (8), the normalized concentration of hormone $\mathrm{c}_{0}$ is subtracted from normalized inflow of hormone $\mathrm{F}_{\mathrm{I}}$. In original Guyton's diagram, the normalized concentration of aldosterone and angiotensin is calculated this way, which means that normalized rate of inflows is $\mathrm{F}_{\mathrm{I}}=\mathrm{AH} \times 0.3333$ and normalized concentration of hormone is $\mathrm{c}_{0}=\mathrm{AHC}$. As a result, AHC is represented by equation (9) instead of equation (8) in original Guyton's diagram. Equation (10) gives the final relation of AHC represented in model:

Correct eq.:

$$
\frac{d(A H C)}{d t}=(A H \times 0.3333-A H C) \times 0.14,
$$

Erroneous eq.:

$$
\frac{d(A H C)}{d t}=A H \times 0.3333-A H C \times 0.14 \text {. }
$$

\section{Model under SIMULINK}

SIMULINK is a block-based language for describing dynamic systems, and also works as a modeling and simulation platform (we used version 7.5.0.342 - R2007b, integrated with MATLAB, The MathWorks, Nattick, MA, USA). It is an interactive and graphic environment dedicated to the multi-domain simulation of hybrid continuous/discrete systems. During simulations, model and block parameters can be modified, and signals can be easily accessed and monitored. In the model, numerical integration was performed using 'ode13t' (a MATLAB library) with a variable step size (maximum step size, auto; relative tolerance, $10 \mathrm{~K} 3$ ).

First, code operations and routines from the computer program were rendered into the SIMULINK graphical description, i.e. elementary blocks and subsystems were connected by appropriate signals and the graphic notation of the original Guyton's diagram was kept as much as possible (Kofránek and Rusz 2007).

Second, subsystems were not treated as 'atomic subunits'. This causes SIMULINK's solver to treat each subsystem as a complete functioning model. Technically, the model works in continuous time and performs all physiological regulations as a complete unit (as the original graphic diagram was designed - the FORTRAN implementation of the model is characterized by a wide range of time scales in the different subsystems), which provides an advantage when designing control systems using principles of complex physiological regulation. All calculations were performed using only the original damping constants obtained from Guyton's diagram.

Finally, to remove a lack of convergence due to oscillation and other run-time errors, the model has addressed the algebraic loops. Note that complex model behavior depends also on correct communication between all subsystems. In this case, it was essential to normalize some of the experimental set and damping constants and supervise model behavior. The complete model is available as open-source on $<$ http://physiome.cz/guyton/>.

\section{Model validation}

In order to validate our corrected SIMULINK implementation of the Guyton's diagram, we simulated four experiments described by Guyton et al. (1972) paper and compared the results with the in vivo data obtained in a series of six dogs (data adopted from Chau et al. 1979), and the original Guyton's model implementation in the FORTRAN environment.

The first experiment is the simulation of hypertension in a salt-loaded, renal-deficient patient by decreasing the functional renal mass to $\sim 30 \%$ of normal and increasing the salt intake to about five times normal on day 0 . This is a very fundamental experiment revealing the importance of the kidneys in blood-pressure control and their influence in the development of essential hypertension (Langston et al. 1963, Douglas et al. 1964, Coleman and Guyton 1969, Cowley and Guyton 1975). The duration of the whole experiment is 12 days.

The second benchmark experiment represents sudden severe muscle exercise and takes place over a much shorter time scale than other experiments ( $5 \mathrm{~min}$ ). The exercise activity was increased to sixty times the normal resting level by setting the exercise activity-ratio with respect to activity at rest after $30 \mathrm{~s}$, corresponding to an approximately 15-fold increase in the whole-body metabolic rate (in this case, the time constant for the local vascular response to metabolic activity was reduced by $1 / 40)$.

The third benchmark experiment simulates the progress of nephrotic edema by increasing seven-fold the rate of plasma-protein loss on day 1 . After seven days, the rate of plasma-protein loss is reduced to three-times above the norm. The duration of the whole experiment is 12 days.

The fourth benchmark experiment simulates the atrioventricular fistula by opening the fistula on day 1 (the constant that represents fistula is set to $5 \%$ ) and closing the fistula on day 5 . The duration of the whole experiment is 9 days.

The goodness-of-fit of the model was also 

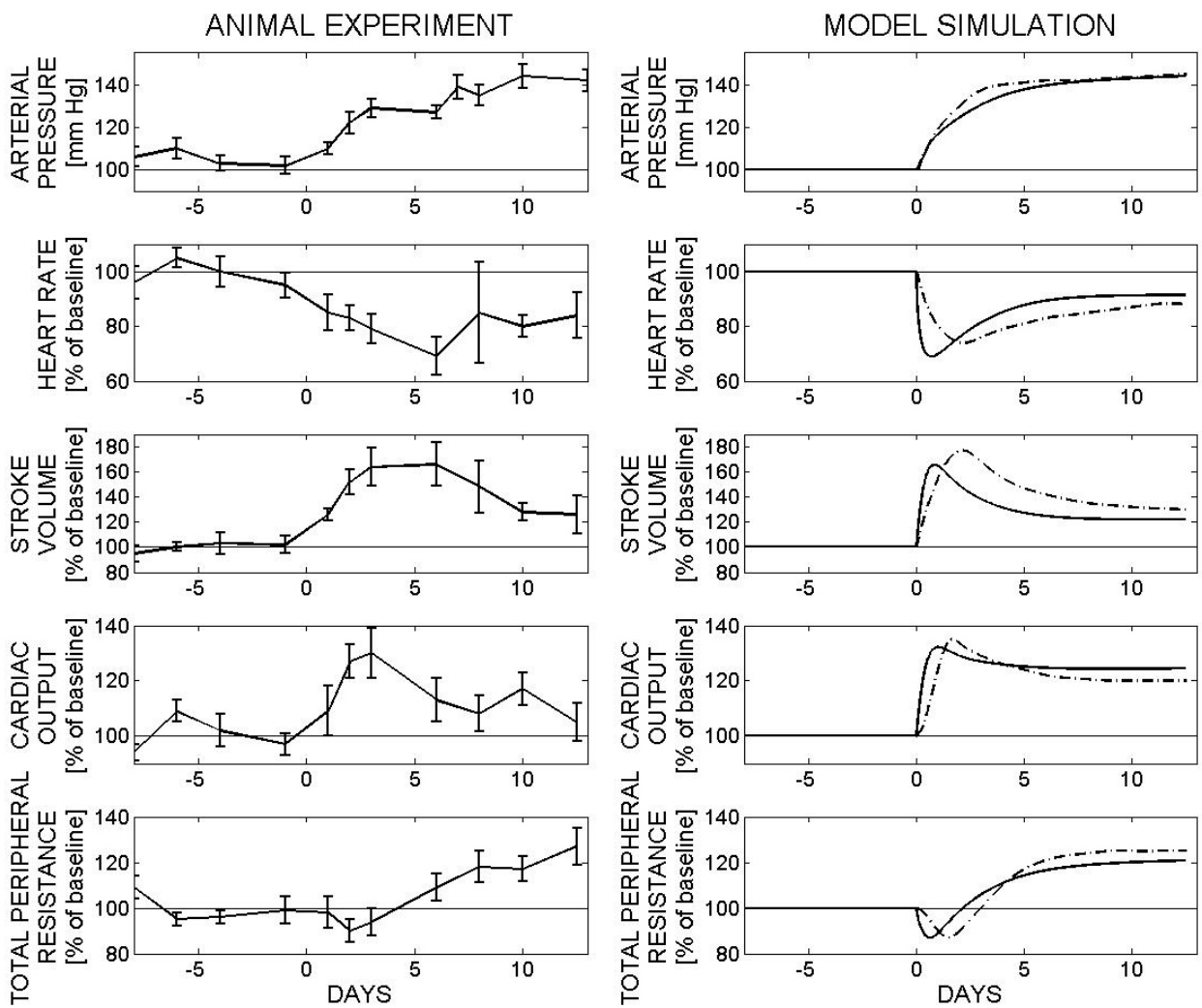

Fig. 3. Simulation of changes in circulatory function at the onset of hypertension caused by reduction of renal mass and an increase in salt intake. Left: Transient changes of different variables in a series of six dogs in which $70 \%$ of the renal mass had been removed and intravenous infusion of saline at a rate of 2 to 3 liters per day was given for 12 days (data adopted from Chau et al. 1979). Right: Model simulation of the same experiment as that seen on the left in dogs; performed by our corrected implementation in SIMULINK (solid lines) in comparison with original Guyton et al. (1972) implementation in FORTRAN (dashdot lines). Changes in all variables are essentially similar to those found in the animals. compared in terms of the chi-square $\left(\chi^{2}\right)$ test between observed simulation results and predicted clinical data.

\section{Results}

Figure 3 represents the results of the simulation of hypertension (Experiment 1). The cardiac output rose at first to $\sim 30 \%$ above normal but then was stabilized by the end of 12 days. The arterial pressure rises more slowly, requiring several days to reach high elevation. During the next days it remained at its new high level indefinitely, as long as the high salt intake was maintained. The simulation is quite sufficient to predict the available data with high statistical significances of $\chi^{2}$ $(11)=1445 ; p<0.001$ for simulation of the arterial pressure, $\chi^{2}(10)=939 ; p<0.001$ for simulation of the heart rate, $\chi^{2}(10)=1388 ; p<0.001$ for simulation of the stroke volume, $\chi^{2}(10)=1189 ; p<0.001$ for simulation of the cardiac output, and $\chi^{2}(10)=1304 ; p<0.001$ for simulation of the total peripheral resistance.

Figure 4 presents the results of the muscle exercise simulation (Experiment 2). At the onset of exercise, cardiac output and muscle blood flow increased considerably and within a very short time. Urinary output fell to its minimal level, while arterial pressure rose moderately. Muscle cell and venous $\mathrm{PO}_{2}$ fell rapidly. Muscle metabolic activity showed an instantaneous increase but then decreased considerably because of the development of a metabolic deficit in the muscles. When exercise was stopped, muscle metabolic activity fell below normal, but cardiac output, muscle blood flow and arterial pressure remained elevated for a while as the person was repaying the oxygen dept.

Figure 5 illustrates the results of the nephrosis simulation (Experiment 3). The principal effect of nephrosis consists of urine protein excretion that may or may not be associated with significant changes in other renal functions. A deficit of the total plasma protein reduces the oncotic pressure, resulting in a fluid redistribution from the blood to the interstitial compartment and an increase of the (mostly free) interstitial fluid volume. Another effect is a mild decrease of cardiac output and arterial pressure. The initial hypoproteinemia only slightly decreased both arterial pressure and cardiac output but induced a notable restriction of the urinary output. Thus, the fluid was retained in the organism causing the interstitial swelling, although the volume of the free interstitial fluid remained relatively unchanged until the interstitial fluid pressure stayed negative. After it reached positive values, an apparent edema occurred with a sharp drop in the arterial pressure. When the rate of renal loss of protein was increased to the point where the liver could increase the plasma protein level, the edema was relieved with high 

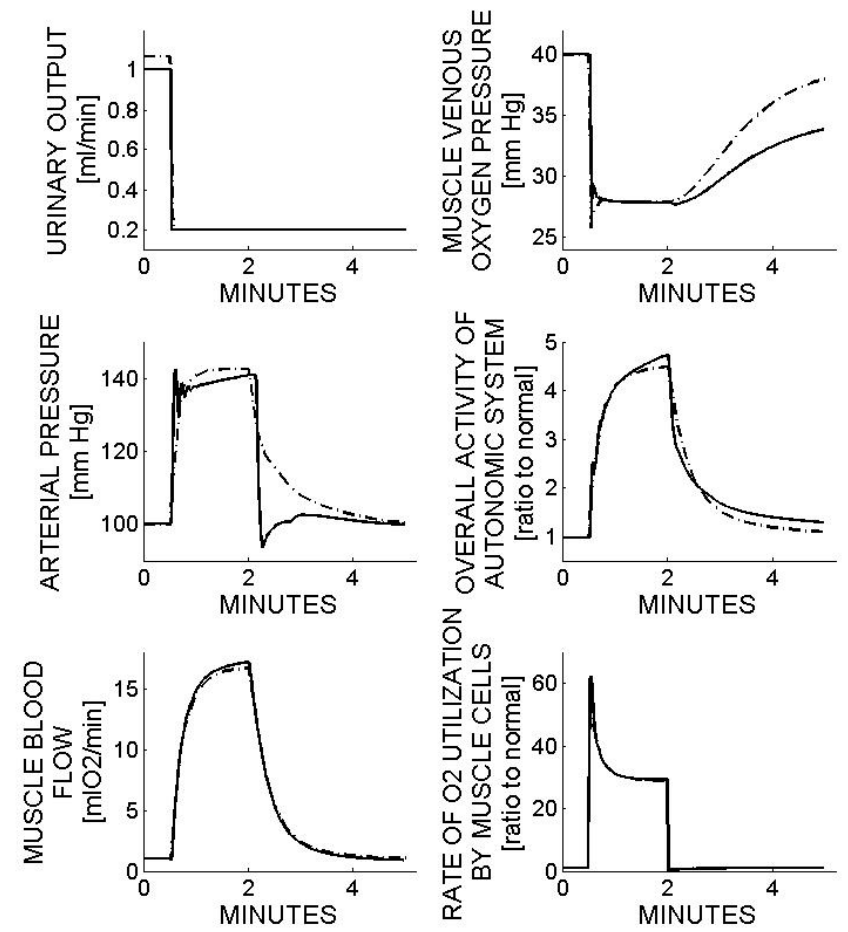

diuresis and increased cardiac output by the end of 12 days.

Figure 6 shows the results of atrioventricular fistula simulation (Experiment 4). Opening the fistula caused an immediate dramatic change in cardiac output, total peripheral resistance and heart rate. Urinary output decreased to minimal threshold levels. As the body adapted, extracellular fluid volume and blood volume increased to compensate for the fistula with the result that after a few days arterial pressure, heart rate and urinary output were near normal levels, while cardiac output doubled and peripheral resistance halved. When the fistula was closed, a dramatic effect occurred with a rapid decrease in cardiac output, rapid increase in peripheral resistance, moderate increase in arterial pressure and moderate decrease in heart rate. Marked diuresis reduced the extracellular fluid volume and blood volume to normal or slightly below. After 9 days, the patient was nearly normal.

\section{Discussion and Conclusion}

The main goal of this paper is the implementation of the core circulatory dynamics model based on Guyton's original diagram and its validation with real experimental data. It was shown how a model might furnish a physiological interpretation for the statistical results obtained on clinical data. We also used the output from Guyton's experiments (Guyton et al.

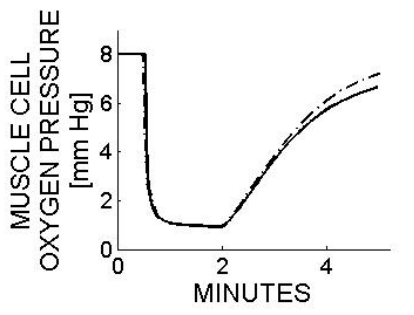

Fig. 4. Benchmark experiment 2: simulation of circulatory dynamics during muscle exercise. At initial break in the curves, the muscles were activated to a level 60 times their normal value. After two minutes, the degree of activation returned to normal. Total experiment time ( $x$-axis) was $5 \mathrm{~min}$. Comparison of simulation results of our SIMULINK model (solid lines) with the original Guyton's model implementation in FORTRAN (dashdot lines).

1972) as a benchmark to validate our implementation. One such problem is the regulation of arterial blood pressure, as was well established by Guyton and his collaborators, since their quantitative systems models led them to a deep reorientation of the understanding of the causes of hypertension (Guyton et al. 1967, Guyton 1980, 1990). This was our rationale for adopting Guyton's diagram as the initial demonstrator of the core model.

As an example of general reflection on physiological regulation, we further discussed the significant differences between the output of the last two simulations including nephrosis and atrioventricular fistula. Both experiments are associated with significant changes in kidneys functions; involving changes in urinary output, arterial pressure, cardiac output, and plasma or blood volume. In simulation of the circulatory changes in nephrosis, the seven-fold rate of plasmaprotein loss caused a fast decrease of proteins volume in the plasma. Reduced oncotic pressure of proteins led to a transfer of water from plasma into interstitium, and a decrease of plasma volume which caused a decrease in arterial pressure. The decreased volume of plasma led also to a decrease of atrial pressure followed by a decrease of the cardiac output. As a result of decreased arterial pressure, vasoconstrictor effects of autonomic autoregulation caused a rapid decrease of urinary output. Reduced volume of plasma proteins lowered the intake of oncotic pressure of proteins in glomerular capillaries, and thus caused an increase in glomerular filtration and 

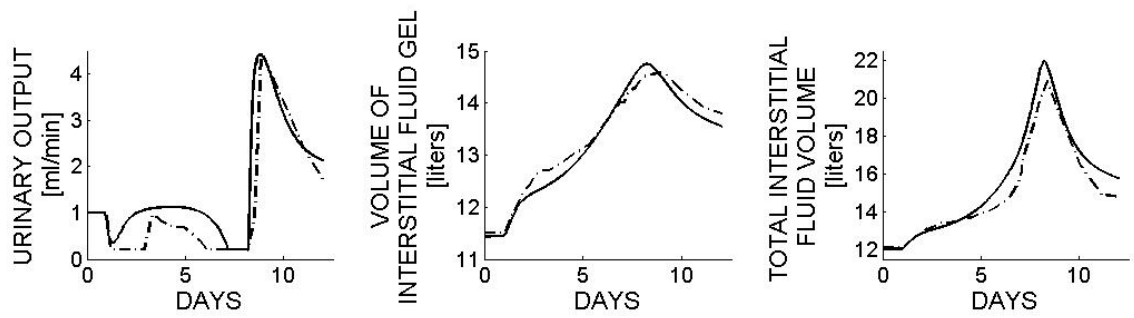

Fig. 5. Benchmark experiment 3 : simulation of circulatory dynamics in nephrosis. At day 1 , the kidneys began to excrete large amount of plasma protein. As a consequence, the fall of the total circulating plasma protein occurred. When the plasma total protein fell below a critical level, an enormous increase in interstitial free fluid occurred. At the end of simulation, an increase in total plasma
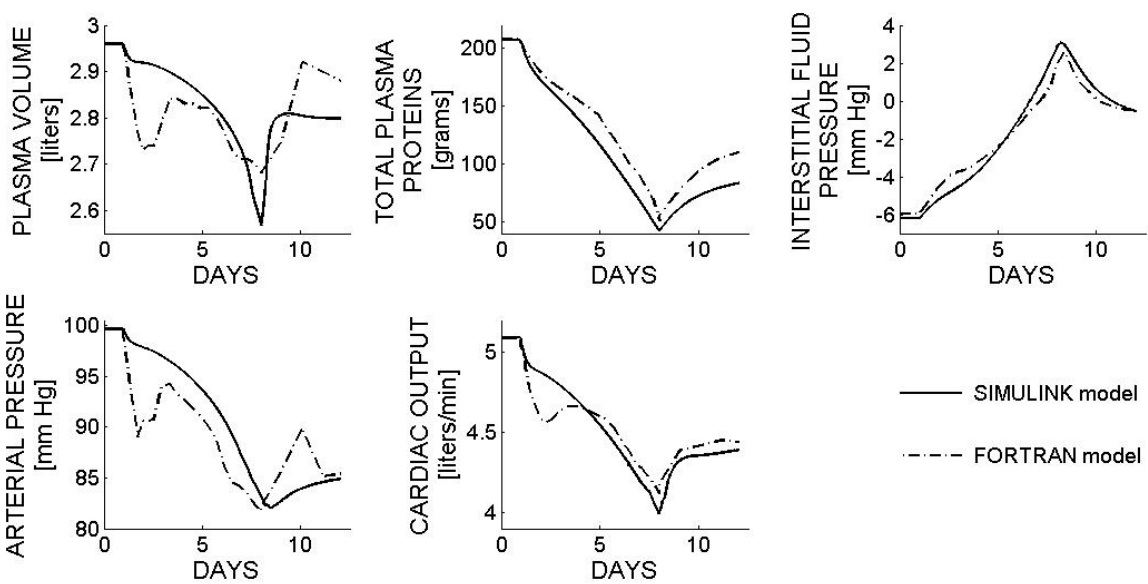
protein caused marked diuresis and beginning resorption of the edema. Total experiment time ( $x$-axis) was 288 hours (12 days). Comparison of simulation results of our SIMULINK model (solid lines) with the original Guyton's model implementation in FORTRAN (dashdot lines).
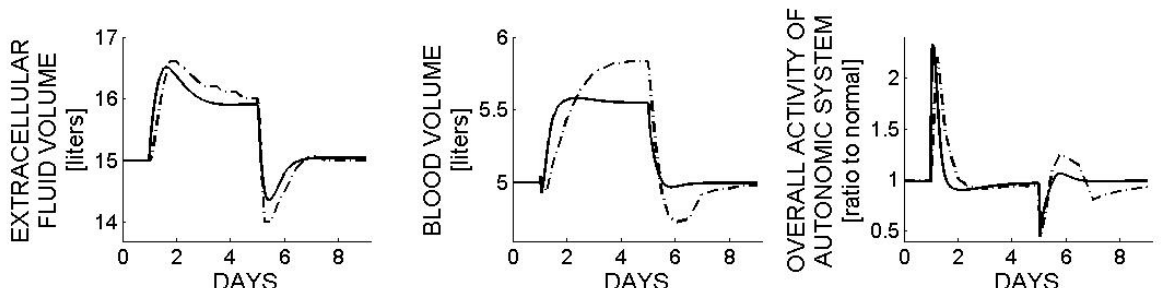

Fig. 6. Benchmark experiment 4: simulation of atrioventricular fistula. At day 1 , the opening of the fistula caused an extreme increase in cardiac output, and decrease in total peripheral resistance. It remains until 5 day where the fistula was closed. At the end of the record, patient was nearly normal. Total experiment time ( $x$-axis) was 9 days. Comparison of simulation results of our SIMULINK model (solid lines) with the original Guyton's model implementation in
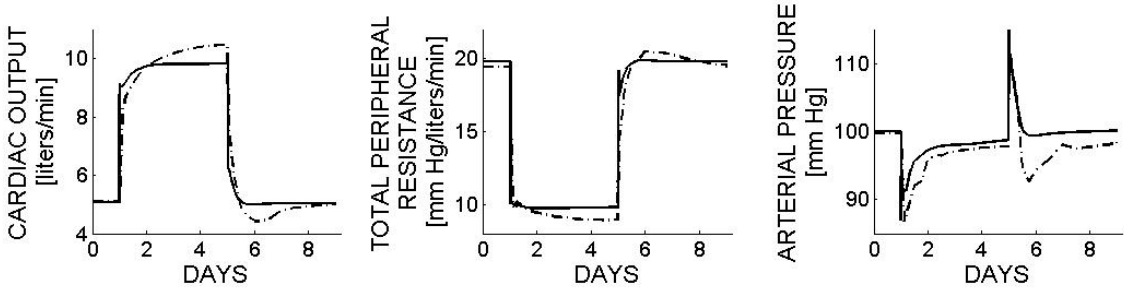

FORTRAN (dashdot lines).
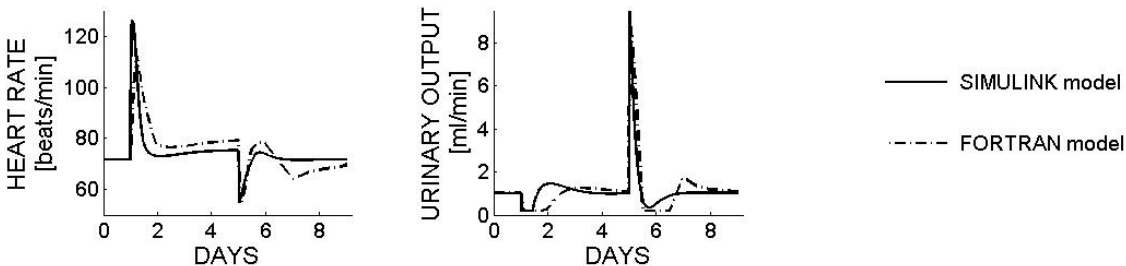

sequential diuresis. Continuous transfer of water from plasma into interstitium and a decrease in arterial pressure resulted in a slow decrease of diuresis into minimal threshold levels. Considering that a simulated patient could not lose more plasma proteins through the kidneys, the rate of plasma protein loss was reduced to three-fold of the norm after 7 days of the experiment. This effect was sufficient to stop the decrease and sequential increase of the concentration of plasma proteins in consequence of protein synthesis progress in the liver. Considering water accumulation in interstitium, the interstitial fluid pressure increased, a slight increase of proteins was sufficient to invert equilibrium on the capillary membrane, and water began to be resorbed from interstitium to plasma. This was associated with increased plasma volume and sequential diuresis. The results from the simulation are almost identical with those that occur in patients with nephrosis (Guyton et al. 1972, Lewis et 
al. 1998). This includes the failure to develop sufficient amounts of edema until the protein concentration falls below a critically low level of about third of normal (Guyton et al. 1972). The simulation also shows the typical tendency of nephrotic patients to have a mild degree of circulatory collapse and slightly decreased plasma volume (Guyton et al. 1972). Another important effect is the changing level of urinary output, a feature that also occurs in nephrotic patients, with urinary output falling very low during those periods where large amounts of edema are being actively formed and the urinary output becoming great during those periods when edema is being resorbed (Guyton et al. 1972).

Similarly as the simulation of nephrosis, the simulation of the atrioventricular fistula was associated with an initial rapid decrease of urinary output. Opening the fistula caused a dramatic decrease of peripheral resistance and an immediate increase of cardiac output. This resulted in acute reaction of autonomic system which rapidly decreased glomerular filtration by increasing of renal vascular resistance, and thus practically stopped the urinary output. As a consequence of the stopped urinary output, the blood volume was increased, vasoconstrictor reaction in kidneys was subsided, and diuresis was re-established. Circulatory system dynamics shifted to its new dynamic equilibrium with increased cardiac output and blood volume, and decreased peripheral resistance. After a closure of the fistula, the whole process was reoriented. The kidneys rapidly excreted redundant blood volume and circulatory dynamics system returned to normal levels. The results from the simulation are almost identical with those that occur during clinical observation of the effects of closing and opening a fistula in animals (Frank et al. 1955). An important effect of fistula management was reported by Friesen et al. (2000). This simulation also shows the essential importance of renal blood volume control for maintenance of blood pressure.

Our circulatory dynamics model can also be used to simulate other experiments including simulations of development of general heart failure, effects of sympathetic nervous system blockade on circulatory function, effect of infusion of different types of substances, effects of vasoconstrictor agents acting on different parts of the circulation, effects of extreme reduction of renal function on circulatory function, and others. Created SIMULINK diagram involves tracking the values of physiological functions during simulation experiments and also disconnect the individual regulation circuits using switches. It allows tracking the importance of individual regulation circuits in progression of a number of various pathological conditions. As an example, in atrioventricular fistula experiment, when the AUM-parameter (sympathetic arterial effect on renal arteries) is returned to its normal value, the kidneys will not respond on increased autonomic system activity. Similarly, in the nephrotic experiment, when the PPCparameter (plasma colloid osmotic pressure) is returned to its normal value, the kidneys will not increase diuresis in response to the decrease of plasma protein volume. The restored Guyton's diagram has become an interactive educational aid that allows through model experiments, a better reflection of general physiological regulations in the pathogenesis of various diseases.

The result of this study is not only a complex functional model, but also a correction of the frequently published Guyton's diagram, which still remains a landmark achievement. The model evolved over the years, but the core of the model and the basic concepts remained untouched and many of the principles contained in the original model have been incorporated by others into advanced models (Abram et al. 2007, Hester et al. 2008). The originality of our core model implementation is our commitment to provide a documentation for each basic module and continuous interactive modification and development of any aspect of the model parameters or equation and its documentation. The complex medicine simulator based on the quantitative physiological model will make it possible to simulate a number of pathological conditions on a virtual patient and the effect of using an artificial organ on normal physiological function can also be simulated. These include artificial heart, artificial ventilator, dialysis, and others.

\section{Conflict of Interest}

There is no conflict of interest.

\section{Acknowledgements}

This research was supported by the research programs "Studies at the molecular and cellular levels in normal and in selected clinically relevant pathologic states" MSM 0021620806, and "Transdisciplinary Research in Biomedical Engineering" MSM 6840770012, and by the grants "e-Golem: medical learning simulator of human physiological functions as a background of e-learning teaching of critical care medicine" MSM 2C06031, „Analysis and Modelling Biological and Speech Signals" GAČR 102/08/H008, and by the Grant Agency of the 
Czech Technical University in Prague FORTRAN implementation of the original Guyton's SGS10/180/OHK3/2T/13, and by Creative Connection model.

Ltd. We are obliged to R. J. White for provision of

\section{References}

ABRAM SR, HODNETT BL, SUMMERS RL, COLEMAN TG, HESTER RL: Quantitative circulatory physiology: an integrative mathematical model of human physiology for medical education. Adv Physiol Educ 31: 202-210, 2007.

BASSINGTHWAIGHTE JB: Strategies for the Physiome Project. Ann Biomed Eng 28: 1043-1058, 2000.

BASSINGTHWAIGHTE J, HUNTER P, NOBLE D: The Cardiac Physiome: perspectives for the future. Exp Physiol 94: 597-605, 2009.

BRUCE NVV, MONTANI J-P: Circulation and fluid volume control. In: Integrative Physiology in the Proteomics and Post-Genomics Age. W WALZ (ed.), Humana Press, Totowa, NJ, 2005, pp 43-66.

CHAU NP, SAFAR ME, LONDON GM, WEISS YA: Essential hypertension: an approach to clinical data by the use of models. Hypertension 1: 86-97, 1979.

COLEMAN TG, GUYTON AC: Hypertension caused by salt loading in the dog. III. Onset transients of cardiac output and other circulatory variables. Circ Res 25: 152-160, 1969.

COWLEY AW, GUYTON AC: Baroreceptor reflex effects on transient and steady-state hemodynamics of salt-loading hypertension in dogs. Circ Res 36: 536-546, 1975.

DOUGLAS BH, GUYTON AC, LANGSTON JB, BISHOP VS: Hypertension caused by salt loading. II. Fluid volume and tissue pressure changes. Am J Physiol 207: 669-671, 1964.

FRANK CV, WANG H-H, LAMMERANT J, MILLER R, WEGRIA R: An experimental study of the immediate hemodynamic adjustments to acute arteriovenous fistulae of various sizes. J Clin Invest 34: 722-731, 1955.

FRIESEN CH, HOWLETT JG, ROSS DB: Traumatic coronary artery fistula management. Ann Thorac Surg 69: 19731982, 2000.

GUYTON AC: Arterial Pressure and Hypertension. Saunders, Philadelphia, PA, 1980.

GUYTON AC: The surprising kidney-fluid mechanism for pressure control - its infinite gain! Hypertension 16: $725-$ $730,1990$.

GUYTON AC, COLEMAN TG: Long-term regulation of the circulation: interrelationships with body fluid volumes. In: Physical Bases of Circulatory Transport Regulation and Exchange. EB REEVE, AC GUYTON (eds), Saunders, Philadelphia, PA, 1967, pp. 179-201.

GUYTON AC, COLEMAN TG, GRANDER HJ: Circulation: overall regulation. Annu Rev Physiol 41: 13-41, 1972.

GUYTON AC, JONES CE, COLEMAN TG: Circulatory Physiology: Cardiac Output and Its Regulation. WB Saunders Company, Philadelphia, 1973, p. 486.

HALL JE: The pioneering use of system analysis to study cardiac output regulation. Am J Physiol 287: R1009-R1001, 2004.

HESTER RL, COLEMAN T, SUMMERS R: A multilevel open source integrative model of human physiology. FASEB J 22: 756.8, 2008.

HUNTER PJ, ROBINS P, NOBLE D: The IUPS Physiome Project. Pflügers Arch 445: 1-9, 2002.

KOFRÁNEK J, RUSZ J: From graphic charts to educational models (in Czech). Cesk Fysiol 56: 69-78, 2007.

LANGSTON JB, GUYTON AC, DOUGLAS BH, DORSETT PE: Effect of changes in salt intake on arterial pressure and renal function in nephrectomized dogs. Circ Res 12: 508-513, 1963.

LEWIS DM, TOOKE JE, BEAMAN M, GAMBLE H, SHORE AC: Peripheral microvascular parameters in the nephrotic syndrome. Kidney Int 54: 1261-1266, 1998.

MANNING RD JR, COLEMAN TG, GUYTON AC, NORMAN RA JR, MCCAA RE: Essential role of mean circulatory filling pressure in salt-induced hypertension. Am J Physiol 236: R40-R47, 1979.

MONTANI J-P, VAN VLIET BN: Understanding the contribution of Guyton's large circulatory model to long-term control of arterial pressure. Exp Physiol 94: 382-388, 2009.

NGUYEN CN, SIMANSKI O, KÄHLER R, SCHUBERT A, JANDA M, BAJORAT J, LAMPE B: The benefits of using Guyton's model in a hypotensive control system. Comput Methods Programs Biomed 89: 153-161, 2008. 
OSBORN JW, AVERINA VA, FINK GD: Current computational models do not reveal the importance of the nervous system in long-term control of arterial pressure. Exp Physiol 94: 389-396, 2009.

SRINISAVAN RS, LEONARD JI, WHITE RJ: Mathematical modelling of physiological states. In: Space Biology and Medicine, CS HUNTOON, VV ANTIPOV, AI GRIGORIEV (eds), vol. 3, American Institute of Aeronautics and Astronautics, Reston, VA, 1996, pp 559-594.

THOMAS SR, BACONNIER P, FONTECAVE J, FRANÇOISE JP, GUILLAUD F, HANNAERT P, HERNÁNDEZ A, LE ROLLE V, MAZIÈRE P, TAHI F, WHITE RJ: SAPHIR: a physiome core model of body fluid homeostasis and blood pressure regulation. Philos Transact A Math Phys Eng Sci 366: 3175-3197, 2008.

WHITE RJ, LEONARD JI, SRINIVASAN RS, CHARLES JB: Mathematical modelling of acute and chronic cardiovascular changes during extended duration orbiter (EDO) flights. Acta Astronaut 23: 41-51, 1991.

WHITE RJ, BASSINGTHWAIGHTE JB, CHARLES JB, KUSHMERICK MJ, NEWMAN DJ: Issues of exploration: human health and wellbeing during a mission to Mars. Adv Space Res 31: 7-16, 2003. 